\title{
A eficácia do ácido tranexâmico tópico no tratamento do melasma: evidências clínicas
}

\author{
Efficacy of topical tranexamic acid in the treatment of melasma: clinical evidence
}

\author{
Marcela Nara Nogueira ${ }^{1 *}$, Lílian de Abreu Ferreira ${ }^{2}$ \\ ${ }^{1}$ Acadêmica do Curso de Farmácia, Faculdade Patos de Minas, Minas Gerais; ${ }^{2}$ Docente Mestre do departamento de \\ Farmácia da Faculdade Patos de Minas. Especialista em Farmacologia Clínica, Saúde Estética e Cosmetologia.
}

\begin{abstract}
Resumo
Introdução: o melasma é uma desordem hiperpigmentar adquirida, crônica, clinicamente caracterizada como máculas acastanhadas com contornos irregulares e limites claros. $O$ tratamento apresenta diferentes modalidades que incluem a eliminação de possíveis fatores causais, como o uso de protetor solar, além de agentes despigmentantes. $O$ ácido tranexâmico, fármaco tradicionalmente antifibrinolítico, tem sido utilizado nas lesões do melasma devido ao seu efeito hipopigmentador e também na prevenção da hiperpigmentação induzida por UV. Objetivo: realizar uma investigação qualitativa bibliográfica acerca do mecanismo de ação do ácido tranexâmico e sua eficácia no tratamento tópico do melasma, baseada em evidências clínicas. Metodologia: foi realizada uma busca nas bases Pubmed e Embase limitada aos últimos 10 anos, na língua inglesa, utilizando os termos 'melasma' e 'tranexamic acid'. Resultados: foram encontrados sete ensaios clínicos que avaliaram a eficácia do ácido tranexâmico em formulações tópicas com concentração variando de 2 a 5\%. O AT exerce seu mecanismo de ação bloqueando reversivelmente a conversão de plasminogênio em plasmina. Como os ativadores de plasminogênio são gerados pelos queratinócitos, o ácido tranexâmico afeta a função dos queratinócitos. Conclusão: o ácido tranexamico mostrou-se seguro e promissor no tratamento de melasma, tanto em comparação ao placebo quanto em relação a outros despigmentantes, sendo bem tolerado e sem ocorrências de reações adversas severas. Contudo, ensaios clinicos controlados e randomizados com avaliação em larga escala e estudos observacionais de longo prazo são necessários para que a terapia a longo prazo e a ação em combinação a outras terapias e outros medicamentos fiquem bem esclarecidos. Palavras-chave: Ácido Tranexâmico. Melanose. Hiperpigmentação.
\end{abstract}

\begin{abstract}
Introduction: melasma is an acquired hyperpigmentation disorder, chronic, clinically characterized as brownish macules with irregular contours and clear boundaries. The treatment presents different modalities that include the elimination of possible causal factors, like the use of sunscreen, besides depigmenting agents. Tranexamic acid, a traditional antifibrinolytic drug, has been used in the lesions of melasma due to its hypopigmenting effect and also in the prevention of UV-induced hyperpigmentation. Objective: to conduct a qualitative bibliographic investigation about the mechanism of action of tranexamic acid and its efficacy in the topical treatment of melasma, based on clinical evidence. Methodology: a search was peformed in the Pubmed database limited to the last 10 years, in the English language, using the terms 'melasma' and 'tranexamic acid'. Results: we found seven clinical trials evaluating the efficacy of tranexamic acid in topical formulations with concentrations ranging from 2 to 5\%. AT exerts its mechanism of action by reversibly blocking the conversion of plasminogen to plasmin. As plasminogen activators are generated by keratinocytes, tranexamic acid affects the function of keratinocytes. Conclusion: tranexamic acid was safe and promising in the treatment of melasma, both compared to placebo as compared to other lighteners, being well tolerated and without incidents of severe adverse reactions. However, controlled and randomized clinical trials with large-scale evaluation and long-term observational studies are necessary for long-term therapy and action in combination with other therapies and other medications can be well understood.
\end{abstract}

Keywords: Tranexamic Acid. Melanosis. Hyperpigmentation.

\section{INTRODUÇÃO}

As melanodermias, entre elas o melasma, são a terceira causa de problemas dermatológicos no Brasil com grande impacto na qualidade de vida dos pacientes. Investimentos na área de desenvolvimento farmacêutico e pesquisa clínica são frequentemente realizados com o

Correspondente/Corresponding: *Marcela Nara Nogueira - End. Rua Tobias Cândido no 404 - Bairro: Centro. CEP: 38700-120-Patos de Minas/ MG. - Tel: (34) 992067820 -E-mail: marcela.nara@hotmail.com foco no tratamento do melasma. Este consiste em uma hiperpigmentação adquirida, bastante comum afetando áreas fotoexpostas (HANDEL; MIOT, L. D. B.; MIOT, H. A., 2014; ISHIY et al., 2014; MIOT et al., 2009).

A etiopatogenia desta dermatose ainda não está totalmente esclarecida, todavia entre os fatores frequentemente envolvidos estão os fatores genéticos, a fotoexposição, gravidez, uso de contraceptivos orais, endocrinopatias, entre outros. De acordo com a composição étnica, fotótipo da pele e a intensidade da exposição ao sol, a prevalência populacional do melasma pode variar 
muito (BAGHERANI, 2015; GEORGE, 2016; HANDEL; MIOT, L.D. B; MIOT, H. A., 2014).

O melasma acomete indivíduos de todas as raças e ambos os sexos, sendo, contudo, nas Américas, mais comum entre mulheres de origem hispânica e brasileira, na faixa de 30 a 55 anos que vivem em áreas onde há maior incidência de radiação solar. Em cerca de $40-50 \%$ dos casos, a doença é desencadeada pela gravidez e aproximadamente $35 \%$ das mulheres que usam anticoncepcionais são acometidas (HANDEL; MIOT, L.D. B.; MIOT, H. A., 2014; MIOT et al., 2009). Ainda, o melasma principalmente os fototipos III a V, os mais melanizados, de acordo com a classificação Fitzpatrick (HANDEL; MIOT, L. D. B.; MIOT, H. A., 2014).

O clima no Brasil e a miscigenação da população favorecem o desenvolvimento do melasma. Em um estudo realizado em Botucatu -São Paulo, foi estimado que cerca de até $35 \%$ das mulheres são afetadas pelo melasma, considerando suas etnias e diferentes regiões onde vivem (ISHIY et al., 2014). A exata incidência populacional do melasma não é ainda conhecida devido as constantes mudanças no tempo de exposição ao sol das pessoas em relação às atividades diárias, incluindo o lazer (HANDEL; MIOT, L. D. B.; MIOT, H. A., 2014).

O tratamento busca melhorar o aspecto da mancha e os resultados dependem da localização da deposição de pigmento e envolve o uso de agentes tópicos despigmentantes entre outras modalidades de tratamento (BAGHERANI, 2015; KIM et al., 2016). O ácido tranexâmico (AT) vem sendo utilizado como agente hipopigmentador tópico nas lesões de melasma, e também na prevenção da pigmentação induzida pela radiação ultravioleta (UV) (KONDOU; OKADA; TOMITA, 2007; NA et al., 2013). E assim, fica evidente a necessidade de avaliação da sua eficácia, bem como o conhecimento do seu mecanismo de ação na terapêutica do melasma. Logo, o AT é uma opção potencialmente nova de tratamento que pode levar a resultados consideravelmente rápidos sem efeitos colaterais significativos e sérios para o melasma (BUDAMAKUNTLA et al., 2013).

Desta forma, o objetivo do estudo foi realizar uma investigação qualitativa e bibliográfica acerca do mecanismo de ação do ácido tranexâmico e sua eficácia no tratamento do melasma, baseada em evidências clínicas.

\section{METODOLOGIA}

Conduziu-se uma busca na literatura nas bases de dados Pubmed e Embase, abrangendo estudos publicados nos últimos 10 anos (2006 a 2016), nas línguas inglesa e portuguesa. Utilizaram-se os termos 'melasma' e 'tranexamic acid' combinados entre si. Foram incluídos no estudo da eficácia do AT, ensaios clínicos randomizados e metanálises de ensaios clínicos que avaliaram o uso tópico do AT em humanos como monoterapia ou em comparação à outros tratamentos tópicos ou placebo. Estudos pertinentes encontrados na lista de referências dos estudos por busca manual, bem como revisões de atualização foram incluídos na revisão integrativa do mecanismo de ação do AT e fisiopatologia do melasma.

\section{DESENVOLVIMENTO MELANOGÊNESE}

Por definição, a melanogênese é a produção de pigmentos de melanina. A melanina é o determinante primário da cor da pele, produzida pelo processo de melanogênese que acontece no interior dos melanossomas contidos nos melanócitos. Os melanócitos na pele são cercados por queratinócitos (um melanócito é cercado por aproximadamente 36 queratinócitos) aos quais são transferidos os pigmentos da melanina (D'MELLO et al., 2016).

A melanogênese pode ser influenciada por fatores genéticos predeterminados, como idade e etnia. Os fatores intrínsecos incluem moléculas secretadas pelos queratinócitos circundantes, fibroblastos, células inflamatórias, neurais ou endócrinas que são afetadas por condições como gravidez e diabetes, enquanto os fatores extrínsecos incluem radiação UV e certos compostos químicos. A estrutura molecular da melanina é adequada para absorver luz ultravioleta (UV) e visível e, portanto, serve como proteção contra a radiação UV da luz solar (D'MELLO et al., 2016; VIDEIRA; MOURA; MAGINA, 2013).

A enzima tirosinase é o elemento crítico para o início da síntese biológica da melanina. A tirosina é oxidada em L-DOPA que é oxidada em dopaquinona. Ambas as reações são realizadas pela tirosinase (MIOT et al., 2009). A partir desse momento, a presença ou ausência de cisteína controlará a via de reação para síntese dos diferentes tipos de melanina, a eumelanina ou a feomelanina. $\mathrm{Na}$ ausência de cisteína, a eumelanina será sintetizada através da conversão da dopaquinona em leucodopacromo e esta em dopacromo. Na etapa seguinte, há duas vias de degradação de dopacromo, uma em que o rearranjo da molécula, forma o 5,6 diidroxiindol e outro rearranjo da molécula em que é formado o 5,6 diidroxiindol-2-ácido carboxílico. Estas reações ocorrem através das proteínas relacionadas à tirosinase, 1 e 2 (TRP-1 e TRP-2, esta última também chamada de dopacromo tautomerase) resultando na formação da eumelanina. Já a feomelanina será sintetizada, a partir da presença da cistéina, que reage com a dopaquinona formando cistenildopa, que é oxidada em intermediários benzotiazínicos, produzindo a feomelanina. Quando os melanossomas estão com grande concentração de pigmento há a transferência para os queratinócitos circundantes, os quais migram para as camadas da epiderme (LEE et al., 2014; SARKAR et al., 2014).

As eumelaninas constituem um pigmento marrom/ preto, alcalino e insolúvel, enquanto as feomelaninas são um pigmento amarelo/vermelho, também alcalino porém solúvel. Uma maior densidade e distribuição de melanina resulta em uma pele mais escura, mas a proporção de eumelanina/feomelanina também contribui para as diferenças observadas na pigmentação da pele. 
Indivíduos com melanócitos que sintetizam mais feomelanina tendem a ter uma pele mais clara, mais propensa a queimaduras solares e com potencial maior de gerar espécies reativas de oxigênio (EROs), aumentando o dano ao DNA e consequentemente contribuindo para os efeitos fototóxicos da radiação UV e aparecimento de neoplasias em comparação a pele que produz mais eumelanina. Já a eumelanina absorve e dispersa a luz UV atenuando sua penetração na pele e reduzindo os efeitos nocivos e assim, indivíduos com maior concentração de eumalanina são menos propensos a queimaduras solares e se bronzeiam mais facilmente (LIN; FISHER, 2007).

\section{MELASMA}

O melasma é uma desordem hiperpigmentar adquirida, crônica, e bastante comum, afetando em maior proporção o sexo feminino e acometendo áreas expostas ao sol, como rosto, pescoço e braços. Clinicamente se apresenta como máculas acastanhadas com contornos irregulares e limites claros (BAGHERANI, 2015; GEORGE, 2016). Pode ocorrer em indivíduos de qualquer etnia, entretanto possui maior prevalência em indivíduos com fototipos mais altos, especialmente aqueles que vivem em áreas tropicais, sob elevada incidência de radiação UV (TAMLER et al., 2009). A etiopatogenia do melasma ainda é desconhecida, porém vários fatores etiológicos foram propostos na literatura, como exposição solar, pré-disposição genética, fatores vasculares, envelhecimento, terapia de reposição hormonal, contraceptivos orais, processos inflamatórios na pele, fármacos fotossensibilizantes, distúrbios endócrinos, stress e gravidez, todos dos quais aumentam significativamente a atividade da tirosinase (GEORGE, 2016; HANDEL; MIOT, L. D. B.; MIOT, H. A., 2014; SARKAR et al., 2014). Além disso, alguns autores relatam a associação entre a melanogênese e a vascularização da pele. Ainda assim, a exposição solar é o principal fator desencadeante para o melasma, estimulando a melanogênese através dos seguintes mecanismos:

- Aumenta a proliferação e atividade de melanócitos;

- Promove a transferência dos pigmentos de melanina para os queratinócitos;

- Estimula os mediadores inflamatórios. A radiação UV provoca a peroxidação de lípidos nas membranas celulares, levando à geração de radicais livres, que induz os melanócitos a produzirem melanina em excesso. Após a exposição aos raios UV, as prostaglandinas (PGs) ativam as vias de sinalização envolvidas no crescimento, diferenciação e apoptose dos melanócitos. A PG E2 é liberada abundantemente pelos queratinócitos após a radiação UV. Isso estimula a formação de dendritos e a da tirosinase;

- Aumenta a expressão dos mediadores melanogênicos como o hormônio estimulante alfa-melanócito (a-MSH). Os queratinócitos respondem à radiação aumentando a expressão do $\alpha-\mathrm{MSH}$ com consequente ativação do MC1R (receptor 1 para melanocortina) presente no melanócito, que leva ao aumenta da expressão do fator de transcrição associado a microftalmia (MITF) aumentando a melanogênese. O MITF regula a transcrição das enzimas tirosinase, TRP - 1 e 2;

- Indução da angiogênese (D’MELLO et al., 2016; GEORGE, 2016; KIM et al., 2016).

$A$ atividade hormonal sexual feminina tem um papel importante na patogênesia do melasma, especialmente nas mulheres. A associação desta condição com a gravidez, pílulas anticoncepcionais orais e hormônios de substituição na pós-menopausa, é bem documentada. Estudos mostraram que a melanogênese é estimulada pelo hormônio luteinizante (LH) e o hormônio folículo-estimulante (FSH). Estes dois ativam o plasminogênio a produzir plasmina (BAGHERANI, 2015). O diagnóstico para melasma é dado inicialmente após observação das desordens pigmentares e exame pela lâmpada de Wood (TAMLER et al., 2009).

Histologicamente, pela lâmpada de Wood, há quatro tipos de melasma. No tipo epidérmico o depósito de melanina fica restrito à camada basal e às camadas acima desta, estendendo-se ocasionalmente até a camada córnea. Na lâmpada de Wood, a pigmentação se acentua, bem como o contraste da cor a medida que a luz é absorvida pela melanina em excesso dessas regiões. Os melasmas mais intensamente vistos na análise da lâmpada de Wood respondem melhor aos tratamentos tópicos (TAMLER et al., 2009; HANDEL; MIOT, L. D. B.; MIOT, H. A., 2014).

$\mathrm{O}$ tipo dérmico consiste naquele onde há pigmentação na epiderme estendendo-se até a derme média, principalmente no interior de melanófagos, e em disposição perivascular. Este tipo também pode envolver a camada mais profunda da derme e não responde bem aos tratamentos. A pigmentação não se acentua na luz de Wood (NICOLAIDOU; KATSAMBAS, 2014; STEINER et al., 2011). No tipo misto, a pigmentação fica mais evidente em algumas áreas, e em outras não há mudança. Já o tipo indeterminado, encontrado nos fototipos V e VI não é bem visualizado à lâmpada de Wood, devido a melanina ser mais abundante nesses fototipos e assim, a maior parte da luz é absorvida. A pele é visualizada escura como um todo (NICOLAIDOU; KATSAMBAS, 2014; TAMLER et al., 2009).

O tratamento terapêutico do melasma apresenta obstáculos na maioria dos casos, uma vez que a condição 
tem tendência a recidivas, está propensa à uma remissão não completa e insatisfatória e hiperpigmentação rebote (GEORGE, 2016; SARKAR et al., 2014;). Diferentes modalidades de tratamento que incluem a eliminação de possíveis fatores causais, como o uso de protetor solar, agentes hipopigmentantes e terapias com laser podem ser utilizadas. Normalmente, os agentes despigmentantes são prescritos em combinação com outras terapias, como a tretinoína, corticosteróides tópicos ou peelings superficiais (EBRAHIMI; NAEINI, 2014). Estudos têm demonstrado que o ácido tranexâmico (AT), fármaco tradicionalmente antifibrinolítico, possui efeito hipopigmentador nas lesões do melasma e também evita a pigmentação induzida por UV (EBRAHIMI; NAEINI, 2014).

\section{ÁCIDO TRANEXÂMICO}

O AT foi relatado primeiramente por Nijo Sadako no ano de 1979, quando utilizava o AT para tratar um paciente com urticária crônica e observou a redução do melasma após um período de duas a três semanas de tratamento. Então, ele realizou o primeiro ensaio clínico com pacientes acometidos pelo melasma. Foi observado que $1,5 \mathrm{~g} / \mathrm{dia}$ de AT oral administrados juntamente com suplementos de vitamina B, C e E por um período de 5 meses teve uma resposta positiva em 11 dos 12 pacientes com idade entre 30 e 69 anos que participaram do estudo (TSE; HUI, 2013).

O AT (trans-ácido 4-amino-metil-ciclohexano carboxílico) é um fármaco hidrofílico, derivado sintético da lisina e exerce seu mecanismo de ação antifibrinolítico através de sua ligação reversível aos sítios da lisina, na molécula de plasminogênio, inibindo o ativador de plasminogênio. Esta inibição bloqueia a conversão do plasminogênio em plasmina, principal agente responsável pela fibrinólise (BAGHERANI, 2015; STEINER et al., 2011).

O plasminogênio está presente também nas células basais epidérmicas. A indução do ativador do plasminogênio pela exposição UV resulta no processo de melanogênese. Além disso, tanto os anticoncepcionais quanto a gravidez ativam esse processo através do aumento do ativador do plasminogênio sérico (BAGHERANI, 2015; STEINER et al., 2011; TSE; HUI, 2013).

O AT também impede a ligação de plasminogênio aos queratinócitos e, assim, inibe a atividade de plasmina induzida por UV. A plasmina é uma protease que melhora a liberação intracelular de ácido araquidônico (AA) e do hormônio alfa estimulador de melanócitos ( $\alpha-M S H)$. $O$ AA e o $\alpha-M S H$ estimulam a melanogênese . Portanto, a atividade antiplasmina do AT é considerada como o mecanismo principal do efeito hipopigmentador desse agente (EBRAHIMI; NAEINI, 2014; GEORGE, 2016).

Há ainda a inibição da produção de prostaglandinas (PGs), com consequente redução da atividade da tirosinase nos melanócitos. Esta característica particular do AT é aplicada com sucesso no tratamento de hiperpigmentações pós-inflamatórias (GEORGE, 2016; KIM et al., 2016).

É discutido na literatura que a radiação UV estimula a produção de fatores angiogênicos, como o fator de crescimento endotelial vascular (VEGF), fator básico de crescimento de fibroblastos (b-FGF) e da interleucina-8. $O$ VEGF interage com os receptores presentes nos queratinócitos epidérmicos que liberam metabolitos de AA e plasminogênio dos vasos proliferados melhorando a melanogênese. A plasmina desempenha um papel importante na liberação do $b-F G F$, que é um potente fator de crescimento de melanócitos e promove sua proliferação. O AT indiretamente reduz a produção de b-FGF e a neovascularização induzida por este e a angiogênese (POOJARY; MINNI, 2015; TSE; HUI, 2013).

O AT não só reduz os níveis de tirosinase, mas também diminui os níveis de TRP-1 e TRP-2. A ativação da via de sinalização induz a degradação do MITF, diminuindo a melanogêses. O MITF é o principal fator de transcrição que rege estas enzimas envolvidas na melanogênese. O AT estimula a via de sinalização e reduz o nível de proteína MITF reduzindo a melanogênese induzida pela inflamação, diminuindo a expressão da tirosinase. O AT também é capaz de suprimir a melanogênese ao regular a transcrição da tirosinase (TSE; HUI, 2013).

Há mais de quatro décadas que $O$ AT é utilizado tanto por via oral como intravenosa como inibidor fibrinolítico. Já como agente despigmentante, o fármaco pode ser administrado por via oral, tópica, intradérmica ou intravenosa. A via tópica tem sido relatada por muitos autores como isenta de efeitos adversos graves (GEORGE, 2016, KANECHORN et al., 2012).

\section{EFICÁCIA CLÍNICA DO ÁCIDO TRANEXÂMICO TÓPICO}

Foram encontrados sete ensaios clínicos que avaliaram a eficácia do ácido tranexâmico em formulações tópicas.

Em um estudo a eficácia de uma formulação tópica de AT a $2 \%$ para o tratamento do melasma foi avaliada. Os escores obtidos apontaram melhoras significativas em 22 dos 23 participantes, após duas semanas de aplicação. Foi observada diminuição significativa pelo Índice de Severidade do Melasma (MASI) sem efeitos colaterais significativos. O MASI consiste em uma medida de resultado desenvolvida para fornecer uma quantificação mais precisa da gravidade do melasma e suas alterações durante a terapia (PANDYA et al., 2011). A melhoria foi prontamente aparente na quarta semana de tratamento, sendo que houve uma melhora gradual durante o restante do período de 12 semanas (KIM et al., 2016).

Em um estudo randomizado, controlado, 15 mulheres receberam quatro sessões mensais de luz intensa pulsada em ambos os lados do rosto. O AT tópico ou o placebo foram aleatoriamente aplicados a um lado do rosto durante e após o tratamento com a luz intensa pulsada. Os pacientes foram acompanhados durante 12 semanas após completar o tratamento. Treze indivíduos completaram o estudo sem eventos adversos graves. Houve melhora significativa após as doze semanas no lado do rosto com 
AT tópico. Os autores concluíram que o AT tópico pode ser considerado um adjuvante eficaz e seguro para tratamento convencional para melasma (CHUNG; LEE; LEE, 2016).

Trinta mulheres com melasma bilateral foram selecionadas em um ensaio cego com duração de 12 semanas para comparar os efeitos terapêuticos do ácido tranexâmico lipossomal e da hidroquinona convencional no melasma. As pacientes aplicaram $5 \%$ de AT lipossomal tópico e $4 \%$ de hidroquinona em creme nos lados designados do rosto duas vezes ao dia, além do protetor solar pela manhã. A pigmentação da pele foi medida usando o MASI em cada visita durante todos os meses do tratamento e até um mês após. Vinte e três pacientes completaram o estudo. Os valores médios de MASI reduziram significativamente nos dois lados tratados $(P<0,001)$ após 12 semanas. Observou-se uma diminuição maior com o AT, embora esta diferença não tenha sido estatisticamente significante. A irritação ocorreu em três pacientes com hidroquinona, enquanto não ocorreram eventos adversos graves com AT (BANIHASHEMI et al., 2015).

O objetivo de um ensaio clínico randomizado duplo cego foi avaliar a eficácia e a segurança da solução tópica a 3\% de AT e compará-la com uma solução de hidroquinona a $3 \%$ combinada com $0,01 \%$ de dexametasona, considerada como tratamento padrão de melasma em mulheres iranianas. Cinquenta pacientes portadoras de melasma aplicaram solução tópica de AT em um lado do rosto e a solução padrão no outro lado duas vezes por dia por 12 semanas. Observou-se uma tendência decrescente significativa no escore MASI de ambos os grupos sem diferença significativa entre eles durante o estudo $(P<0,05)$. Não foram observadas diferenças na satisfação dos pacientes entre dois grupos $(P<0,05)$. No entanto, os efeitos colaterais da hidroquinona + dexametasona foram significativamente proeminentes em comparação com o AT $(P=0,01)$. Os resultados deste estudo indicam o AT tópico como opção de tratamento eficaz e seguro para o melasma (EBRAHIMI; NAEINI, 2014).

Em outro estudo clínico, foram avaliadas 25 mulheres por 8 semanas para elucidar os efeitos do AT oral e tópico no melasma. As voluntárias foram instruídas a tomar dois comprimidos de AT três vezes por dia e aplicar o AT tópico duas vezes ao dia. A pigmentação da pele e o eritema foram medidos durante cada visita e biópsias cutâneas foram coletadas de oito indivíduos antes e após o tratamento. Vinte e dois indivíduos completaram o estudo e não ocorreram efeitos adversos graves durante o período de estudo. Os scores de MASI reduziram significativamente. A análise histológica mostrou redução significativa da pigmentação epidérmica, dos números dos vasos e da contagem de mastócitos (NA et al., 2013).

Kanechorn et al. (2012) realizaram um ensaio clínico randomizado duplo-cego entre pacientes asiáticos com melasma epidérmico, utilizando $5 \%$ de AT lipossomado em uma formulação de gel por uma duração de 12 semanas em comparação com o veículo em um teste de dupla face. Os pacientes foram orientados a utilizar as formulações duas vezes ao dia, além de um protetor solar escolhido pela manhã. Do total, $78,2 \%$ dos pacientes apresentaram uma diminuição no MASI com clareamento da pigmentação, porém sem significância estatística ( $p$ $>0.05$ ). Entretanto os pacientes apresentaram eritema com significancia estatística no lado da aplicação do AT $(p<0.05)$ (KANECHORN et al., 2012).

Em outro estudo, Kondou, Okada e Tomita (2007) observaram que em 33 pacientes avaliados, uma emulsão contendo $2 \%$ de AT apresentou melhoras acentuadas para o melasma em $80 \%$ dos pacientes após 8 semanas e em $75 \%$ dos indivíduos com sardas, após 12 semanas. Portanto, a melhora exige pelo menos dois meses de aplicação tópica. Os resultados mostraram não apenas o efeito clareador do AT tópico através da inibição da síntese de melanina, como também o efeito preventivo no aparecimento de novas pigmentações (KONDOU; OKADA; TOMITA, 2007).

Embora permaneçam desconhecidas quais as principais causas de melasma, o AT pode atuar promovendo a redução de fatores dérmicos relacionados ao melasma. O AT pode ser usado como agente terapêutico potencialmente seguro, eficaz e promissor para o tratamento do melasma, tendo em vista que a medicação é facilmente disponível e acessível, com resultados relativamente rápidos, sem efeitos colaterais significativos e quase sem tempo de inatividade (BUDAMAKUNTLA et al., 2013).

\section{CONCLUSÃO}

A concentração do ácido tranexâmico nas formulações tópicas variou de 2 a $5 \%$ nos estudos. Do total, em apenas um estudo não foi demonstrada uma eficácia significativa do ácido tranexamico. No entanto, é possível que o efeito momentâneo da proteção solar ou tamanho da amostra tenha sido pequeno para apresentar diferenças estatisticamente significativas entre o AT e seu veículo. $\mathrm{O}$ ácido tranexamico mostrou-se seguro e promissor no tratamento de melasma, sendo bem tolerado e tão efetivo quanto a hidroquinona sozinha ou em associação a dexametasona, sem reações adversas severas. Estudos controlados randomizados com avaliação em larga escala e estudos observacionais de longo prazo ainda são necessários para o futuro, para que fiquem bem documentados a freqüência de uso, benefícios em longo prazo e ação em combinação a outras terapias e outros medicamentos.

\section{REFERÊNCIAS}

BAGHERANI, N. The efficacy of tranexamic acid in the treatment of melasma. Dermatol. Ther., Khorramshahr, v. 28, n. 4, p. 265, 2015.

BANIHASHEMI, M. et al. Comparison of therapeutic effects of liposomal tranexamic acid and conventional hydroquinone on melasma. J. Cosmet. Dermatol., Oxford, v. 14, n. 3, p. 174-177, 2015.

BUDAMAKUNTLA, L. et al. A randomised, open-label, comparative study of tranexamic acid microinjections and tranexamic acid with microneedling in patients with melasma. J. Cutan. Aesthet. Surg., Bangalore, v. 6, n. 3, p. 139-143, 2013. 
CHUNG, J. Y.; LEE, J. H.; LEE, J. H. Topical tranexamic acid as an adjuvant treatment in melasma: Side-by-side comparison clinical study. J. Derm. Treat., Basingstoke, v. 27, n. 4, p. 373-377, 2016.

D’MELLO, S. A. N. et al. Signaling Pathways in Melanogenesis. Int. J. Mol. Sci., Auckland, v. 17, n. 7, p. 1-18, 2016.

EBRAHIMI, B.; NAEINI, F. F. Topical tranexamic acid as a promising treatment for melasma. J. Res. Med. Sci., Bethesda, v. 19, n. 8, p. 753757, 2014.

GEORGE, A. Tranexamic acid: an emerging depigmenting agent. Pig. Intern., Bangalore, v. 3, n. 2, p. 66-71, 2016.

HANDEL, A. C.; MIOT, L. D. B.; MIOT, H. A. Melasma: uma avaliação clínica e epidemiológica. An. Bras. Dermatol., Rio de Janeiro, v. 89, n. 5, p. 771-782, 2014.

ISHIY, P. S. et al. Skin diseases reported by workers from UNESP campus at Rubião Jr, Botucatu-SP (Brazil). An. Bras. Dermatol., Rio de Janeiro, v. 89, n. 3, p. 529-531, 2014.

KANECHORN, N. P. et al. Topical $5 \%$ tranexamic acid for the treatment of melasma in Asians: a double-blind randomized controlled clinical trial. J. Cosmet. Laser. Ther., London, v. 14, n. 3, p. 150-154, 2012.

$\mathrm{KIM}$, S. J. et al. Efficacy and possible mechanisms of topical tranexamic acid in melasma. Clin. Exp. Dermatol., Oxford, v. 41, n. 5, p. 480-485, 2016.

KONDOU, S.; OKADA, Y.; TOMITA, Y. Clinical study of effect of tranexamic acid emulsion on melasma and freckles. Skin res. technol., Copenhagen, v. 6, p. 309-315, 2007.

LEE, M. C. et al. Treatment of melasma with mixed parameters of 1,064$\mathrm{nm} \mathrm{Q}$-switched Nd: YAG laser toning and an enhanced effect of ultrasonic application of vitamin C: a split-face study. Lasers Med. Sci., London, v. 30, n. 1, p. 159-163, 2014.

LIN, J. Y.; FISHER, D. E. Melanocyte biology and skin pigmentation. Na- ture, London, v. 445, n. 7130, p. 843-850, 2007.

MIOT, L. D. B. et al . Fisiopatologia do melasma. An. Bras. Dermatol., Rio de Janeiro, v. 84, n. 6, p. 623-635, 2009.

NA, J. I. et al. Effect of tranexamic acid on melasma: a clinical trial with histological evaluation. J. Eur. Acad. Dermatol. Venereol., Amsterdam, v. 27, n. 8, p. 1035-1039, 2013.

NICOLAIDOU, E.; KATSAMBAS, A. D. Pigmentation disorders: hyperpigmentation and hypopigmentation. Clin. Dermatol., Philadelphia, v. 32, n. 1, p. 66-72, 2014.

PANDYA, A. G. et al. Reliability assessment and validation of the Melasma Area and Severity Index (MASI) and a new modified MASI scoring method. J. Am. Acad. Dermatol., St. Louis, v. 64, n.1, p. 78-83, 2011.

POOJARY, S.; MINNI, K. Tranexamic acid in melasma: a review. Journal of Pigmentary Disorder, [S.I], v. 2, n. 12, p. 1-4, 2015.

SARKAR, R. et al. Melasma update. Indian Dermatol. Online J., Nova Deli, v. 5, n. 4, p. 426-435, 2014.

STEINER, D. et al. Melasma e laser fracionado não ablativo (1540nm): um estudo prospectivo. Surg. Cosm. Dermatol., Rio de Janeiro, v. 3, n. 1, p. 37-40, 2011.

TAMLER, C. et al. Classificação do melasma pela dermatoscopia: estudo comparativo com lâmpada de Wood. Surg. Cosm. Dermatol., Rio de Janeiro, v. 1, n. 3, p. 115-119,

2009.

TSE, T. W.; HUI, E. Tranexamic acid: an important adjuvant in the treatment of melasma. J. Cosmet. Dermatol., Oxford, v. 12, n. 1, p. 57-66, 2013.

VIDEIRA, I. F. S.; MOURA, D. F. L.; MAGINA, S. Mechanisms regulating melanogenesis. An. Bras. Dermatol., Rio de Janeiro, v. 88, n. 1, p. 7683, 2013.

Submetido em: 10/09/2017

Aceito em: 18/12/2017 